\title{
Inhibition by caffeic acid of the influenza $A$ virus multiplication in vitro
}

\author{
HIROTOSHI UTSUNOMIYA ${ }^{1}$, MASAO ICHINOSE ${ }^{1}$, KEIKO IKEDA ${ }^{2,3}$, MISAO UOZAKI ${ }^{3,4}$, JUNKO MORISHITA ${ }^{5,6}$, \\ TOMOMI KUWAHARA ${ }^{5}$, A. HAJIME KOYAMA ${ }^{3,6}$ and HISASHI YAMASAKI ${ }^{3}$ \\ ${ }^{1}$ Department of Strategic Surveillance for Functional Food and Comprehensive Traditional Medicine, \\ ${ }^{2}$ School of Nursing and Health Science, ${ }^{3}$ Division of Virology, Department of Cellular and Molecular Medicine, \\ Wakayama Medical University Graduate School of Medicine, Wakayama 641-8509; ${ }^{4}$ Japanese Red Cross, \\ Wakayama Medical Center, Wakayama 640-8558; ${ }^{5}$ Department of Microbiology, Faculty of Medicine, \\ Kagawa University, Kagawa 761-0793; ${ }^{6}$ Wakayama Shin-ai Women's Junior College, Wakayama 640-0341, Japan
}

Received February 1, 2014; Accepted July 4, 2014

DOI: $10.3892 /$ ijmm.2014.1859

\begin{abstract}
Caffeic acid has been shown to inhibit the multiplication of influenza A virus in vitro, whereas caffeine, quinic acid and chlorogenic acid do not. Caffeic acid has also been shown to have antiviral activity against herpes simplex virus (DNA virus) and polio virus (RNA virus). In the present study, a comparison of the one-step growth curve of the influenza virus in the presence of caffeic acid with that in the absence of the reagent showed that an eclipse period of the virus multiplication in the infected cells was not affected by the reagent, while the progeny virus yield was markedly decreased in the presence of caffeic acid. In additional experiments, it was found that the addition of caffeic acid at an early time point post-infection (within $3 \mathrm{~h}$ post-infection) was mandatory for extensive antiviral activity, suggesting that a major target of the reagent exists in the early stages of infection. Simultaneously with the decrease in the progeny virus yield, both the virusinduced cytopathic effects and apoptotic nuclear fragmentation were markedly suppressed by the reagent, suggesting that caffeic acid suppresses, at least temporally, the degeneration of the virus-infected cells and that the observed antiviral activity is likely not the secondary result of the cytotoxic effects of the reagent. These results suggest the potential pharmacological use of caffeic acid or its derivatives as an antiviral drug against influenza A virus.
\end{abstract}

Correspondence to: Dr Hisashi Yamasaki, Division of Virology, Department of Cellular and Molecular Medicine, Wakayama Medical University Graduate School of Medicine, 580 Mikazura, Wakayama 641-8509, Japan

E-mail: hisay@wakayama-med.ac.jp

Dr A. Hajime Koyama, Wakayama Shin-Ai Women's Junior College, 702-2 Souzaka, Wakayama 640-0341, Japan

E-mail:koyama@shinai-u.ac.jp

Key words: influenza virus, caffeic acid, antiviral, virucidal, apoptosis

\section{Introduction}

The control of influenza A virus infection poses a major social and economical issue. Although neuraminidase inhibitors have been used as an effective treatment for influenza infection, the rapid development of drug-resistant variants of the virus often limits the applicability of these antiviral drugs with single targets. Thus, there is a constant need for the development of novel anti-influenza drugs. Considering the potential safety of natural dietary products, in previous studies, we examined the antiviral and virucidal activities of various natural products and their components (1-3, reviewed in ref. 4). We found that caffeic acid inhibits the multiplication of herpes simplex virus type 1 (HSV-1) in vitro by interfering mainly with the early stages of HSV-1 multiplication in the infected cells prior to the completion of viral genome DNA replication, independently of its cytotoxic action (5).

Caffeic acid (3,4-dihydroxycinnamic acid) is one of the abundant plant-derived polyphenol compounds with 2 phenolic hydroxyl groups and is known to have antioxidant activity (6). This compound is the major metabolite produced by the hydrolyzation of chlorogenic acid, which is commonly found in a variety of foods, including coffee, fruits, vegetables and grains. In the present study, we aimed to further characterize the antiviral activities of caffeic acid against a variety of viruses with different replication mechanisms. We found that the reagent effectively inhibited the multiplication of influenza A virus in MDCK cells, as well as the virus-induced cytopathic effect (CPE). We present the results of an in vitro virological characterization of the antiviral activity of caffeic acid against influenza A virus.

\section{Materials and methods}

Cells and viruses. The MDCK cells (obtained from Dr Katsuhisa Nakajima, Nagoya City University, Nagoya, Japan), HEp-2 cells (obtained from Dr Takahiro Uchida, Tokushima University, Tokushima, Japan) and Vero cells (obtained from Dr Kamesaburo Yoshino, University of Tokyo, Tokyo, Japan) were grown in Eagle's minimum essential medium (MEM) 
(Nissui Pharmaceutical Co., Ltd., Tokyo, Japan) containing 5\% fetal bovine serum (FBS) (Euroclone, Pero, Italy). Influenza virus A/Aichi/68 (H3N2) (obtained from Dr Katsuhisa Nakajima, Nagoya City University), Sabin strain of poliovirus type 1 (PV-1) (from Dr Akio Nomoto, University of Tokyo) and HSV-1 strain F (from Dr Bernard Roizman, Chicago University, Chicago, IL, USA), were used throughout the experiments. The influenza virus was propagated in the MDCK cells in MEM supplemented with both $0.1 \%$ bovine serum albumin (BSA) (Wako Pure Chemical Industries, Ltd., Osaka, Japan) and acetylated trypsin $(4 \mu \mathrm{g} / \mathrm{ml})$ and stored at $-80^{\circ} \mathrm{C}$ until use. The amounts of the viruses were measured by a plaque assay as previously described (7-9). Briefly, Vero (for PV-1 and HSV-1) or MDCK (for influenza virus) cell monolayers were washed once with Dulbecco's phosphate-buffered saline without $\mathrm{Ca}^{++}$ and $\mathrm{Mg}^{++}$(PBS) and received an aliquot of the virus suspension in PBS containing $0.5 \%$ FBS (for PV-1 and HSV-1) or $0.1 \%$ BSA (for influenza virus), followed by an incubation for $60 \mathrm{~min}$ at room temperature with mechanical rocking on a rocker platform (Taitec Co., Ltd., Koshigaya, Saitama, Japan). The infected cell monolayer were incubated at $37^{\circ} \mathrm{C}$ (for HSV-1 and influenza virus) or at $35.5^{\circ} \mathrm{C}$ (for PV-1) in MEM containing $0.5 \% \mathrm{FBS}$ and $0.68 \%$ methylcellulose (Nacalai Tesque Inc., Kyoto, Japan) for PV-1 and HSV-1 or in MEM containing 0.6\% Difco agar noble (Becton, Dickinson and Co., Sparks, MD, USA) and $5.3 \mu \mathrm{g} / \mathrm{ml}$ acetylated trypsin (Sigma-Aldrich Corp., St. Louis, MO, USA) for influenza virus until the formation of plaques.

Effect of the reagent on the virus yields. Caffeic acid, quinic acid and chlorogenic acid were obtained from Wako Pure Chemical Industiries, Ltd. (Osaka, Japan). The reagent solution (1.0 or $100 \mathrm{mM}$ ) was prepared by dissolving the reagents in hot water and neutralizing its acidity with $1 \mathrm{~N}$ sodium hydroxide solution, followed by filtration through a Millipore Dimex membrane (pore size $0.22 \mu \mathrm{m}$; Merck Millipore, Billerica, MA, USA).

Monolayered cells in $35 \mathrm{~mm}$-dishes were infected with the viruses at an indicated multiplicity of infection (MOI). The infected cells were further incubated at $37^{\circ} \mathrm{C}$ (for influenza virus and $\mathrm{HSV}-1$ ) or $35.5^{\circ} \mathrm{C}$ (for PV-1) for the indicated periods of time in serum-free MEM containing both $0.1 \%$ BSA and the indicated concentrations of the reagent. For the influenza virus, acetylated trypsin was added to the infected culture and, at the indicated time point, the culture medium of the infected cells was harvested and the amount of the progeny virus in the medium was determined by a plaque assay as previously described (8).

Determination of CPE and apoptotic cell nuclei. Confluent monolayers of MDCK cells were mock-infected or infected with the influenza virus and were then incubated at $37^{\circ} \mathrm{C}$ for the indicated periods of time in serum-free MEM containing $0.1 \%$ BSA and acetylated trypsin, supplemented with the indicated concentrations of the reagent. The CPE was determined by a microscopic observation of the cells; approximate amounts of rounded cells on cell monolayers were estimated under a phase-contrast microscope (Eclipse E800 model; Nikon Corp., Tokyo, Japan).

Apoptotic cell nuclei were observed after the cells were fixed with methyl alcohol/acetic acid (3:1) and stained for $10 \mathrm{~min}$ with the DNA-binding dye, Hoechst $33258(0.05 \mu \mathrm{g} / \mathrm{ml})$, according to the method previously described in the study by McGarrity (10).

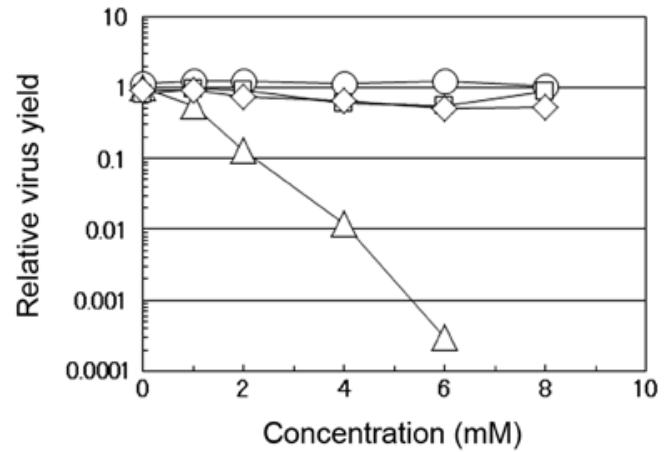

Figure 1. Effect of caffeic acid on the multiplication of influenza virus. Confluent monolayers of MDCK cells were infected with influenza A virus at a multiplicity of infection (MOI) of 9 . The infected cells were incubated at $37^{\circ} \mathrm{C}$ for $12 \mathrm{~h}$ in medium containing $0.1 \%$ bovine serum albumin (BSA) and acetylated trypsin, supplemented additionally with the indicated concentrations of caffeic acid, chlorogenic acid, quinic acid or caffeine. At the end of infection, the amounts of infectious progeny viruses were determined and normalized to the virus yield in the absence of the reagent. $\bigcirc$, caffeine; $\Delta$, caffeic acid; $\square$, quinic acid; $\diamond$, chlorogenic acid.

\section{Results and Discussion}

Effects of caffeic acid on the multiplication of influenza $A$ virus. As shown in Fig. 1, we compared the effects of caffeic acid, caffeine, quinic acid and chlorogenic acid on the multiplication of influenza A virus in the MDCK cells. Chlorogenic acid is an esterified form of caffeic acid with quinic acid and is one of the most widely consumed polyphenols abundant in dietary foods, particularly in coffee. When the virus was propagated in the presence of the indicated concentrations of these compounds, the virus yield at $12 \mathrm{~h}$ post-infection (p.i.) was compared to the yield in the absence of the compounds. The yield markedly decreased with the increasing concentrations of caffeic acid, but not with any of the other 3 compounds. At a dose of $4 \mathrm{mM}$ caffeic acid, the yield of influenza $A$ virus was approximately 100-fold lower than that in the absence of the reagent, while, even at higher concentrations (6 or $8 \mathrm{mM})$, caffeine, quinic acid or chlorogenic acid had insignificant effects on the virus yields. Although caffeine did not suppress the multiplication of influenza A virus even at $8 \mathrm{mM}$ (Fig. 1, cirle), we have previously found that both caffeic acid and caffeine, but neither chlorogenic acid nor quinic acid, inhibit the multiplication of HSV-1 in HEp-2 cells (5), indicating that caffeic acid suppresses the multiplication of both DNA and RNA viruses, in contrast to the selective antiviral activity of caffeine which is effective only against HSV-1 (11).

In addition, in conjunction with the decrease in the virus yield in the presence of caffeic acid, the virus-induced CPE of the infected cells was also suppressed; although not completely, the cell rounding and ballooning, as well as the detachment from the dish surface in the influenza virus-infected cells in culture were significantly suppressed even at $12 \mathrm{~h}$ p.i. in the presence of caffeic acid at $4 \mathrm{mM}$. No such suppression of CPE was observed with the other 3 compounds (data not shown).

The antiviral effects of caffeic acid against influenza A virus were compared with those against other DNA and RNA viruses (i.e., HSV-1 and PV-1, respectively). These 3 viruses have a different genome structure and replication strategy in the infected cells; HSV-1 has a double-stranded DNA genome which 


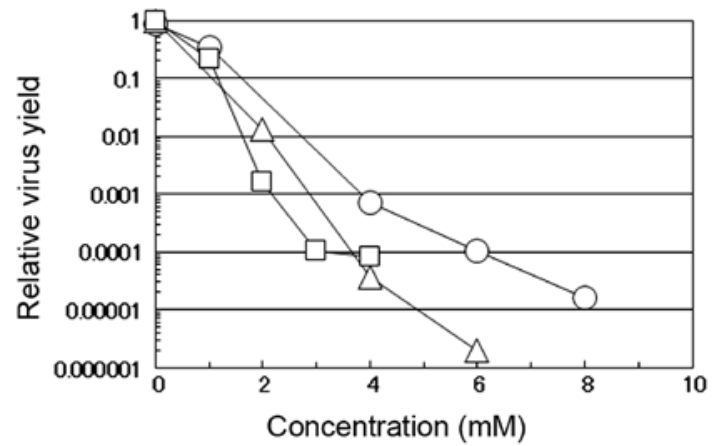

Figure 2. Comparison of antiviral activity of caffeic acid against DNA and RNA viruses. Confluent monolayers of HEp-2 (for HSV-1 and PV-1) or MDCK (for influenza A virus) cells were infected with each of these viruses at a multiplicity of infection (MOI) of 10 for HSV-1, 9 for influenza virus and 5 for PV-1. The infected cells were incubated overnight in medium containing the indicated concentrations of caffeic acid at $37^{\circ} \mathrm{C}$ for HSV-1 and influenza A virus or at $35.5^{\circ} \mathrm{C}$ for PV-1. At the end of infection, the amounts of infectious progeny viruses were determined and normalized to the virus yield in the absence of the reagent. $\bigcirc, \mathrm{HSV}-1 ; \triangle$, influenza A virus; $\square, \mathrm{PV}-1$.

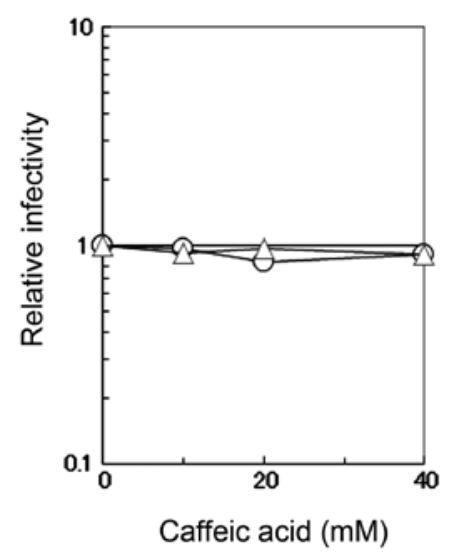

Figure 3. Effect of caffeic acid on the infectivity of influenza A virus. The virus was incubated on ice for $60 \mathrm{~min}$ in $\mathrm{PBS}$ ( $\mathrm{pH} 7.4$ ) containing $0.1 \%$ bovine serum albumin (BSA) and the indicated concentrations of caffeic acid (O) or caffeine $(\triangle)$. Following incubation, the amounts of infectious viruses in PBS were determined and normalized to those in the absence of the reagent.

replicates in the nucleus (12) and PV-1 has a positive-stranded RNA genome which replicates in the cytoplasm (13), while influenza A virus has a negative-stranded RNA genome which replicates in the nucleus of the infected cells (14). When the PV-1-infected or HSV-1-infected HEp-2 cells or influenza virusinfected MDCK cells were incubated in the medium-containing various concentrations of caffeic acid, the yield of the respective virus markedly decreased in a concentration-dependent manner; these effects were more prominent for PV-1 and influenza A virus than for HSV-1 (Fig. 2). These results confirm that caffeic acid can inhibit both DNA and RNA viruses regardless of cell type and suggest that RNA viruses are more sensitive to the reagent than DNA viruses. The results also indicate that both enveloped (influenza virus and HSV-1) and non-enveloped (PV-1) viruses are sensitive to caffeic acid. It may be noteworthy that, although PV-1 induced massive CPE in the infected HEp-2 cells, CPE induced by PV-1 infection was also markedly suppressed by the addition of caffeic acid (data not shown), similar to the effects observed by infection with the influenza virus.

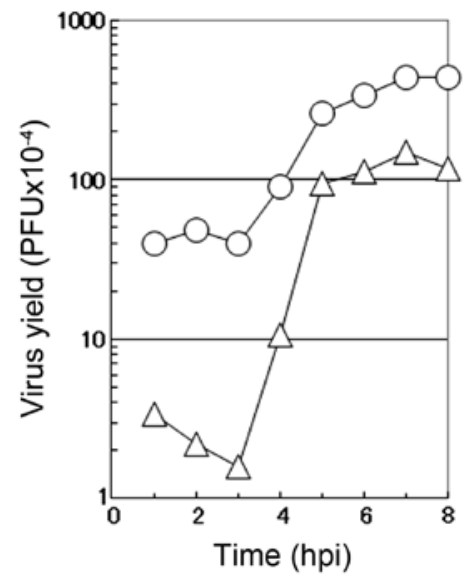

Figure 4. Effect of caffeic acid on the one-step growth curve of influenza A virus. Confluent monolayers of MDCK cells were infected with influenza A virus at a multiplicity of infection (MOI) of 7. The infected cells were incubated at $37^{\circ} \mathrm{C}$ for $8 \mathrm{~h}$ in Eagle's minimum essential medium (MEM) containing $0.1 \%$ bovine serum albumin (BSA) and acetylated trypsin supplemented with $(\triangle)$ or without (O) $2.0 \mathrm{mM}$ caffeic acid. At the indicated time point, the amounts of infectious progeny viruses in the culture medium were determined.

Effects of caffeic acid on virus infectivity and cell viability. The direct effects of caffeic acid on the infectivity of influenza A virus were measured by incubating the virus in PBS containing $0.1 \%$ BSA and various concentrations of caffeic acid or caffeine. The viral infectivity was not affected by caffeic acid or caffeine even at the concentration of $40 \mathrm{mM}$ (Fig. 3). This concentration is much higher than that used for the antiviral assay described above (Figs. 1 and 2), indicating that the observed antiviral activity is not a result of the virucidal effects of caffeic acid.

In addition, although we have previously found (5) that caffeic acid has significant cytotoxic activity during the prolonged incubation of cells $(>24 \mathrm{~h})$, the cytotoxicity was mild and not sufficiently strong (data not shown) to explain the observed antiviral effect, particularly by a short incubation period for the antiviral assay against influenza virus $(<12 \mathrm{~h})$. The suppression by caffeic acid of CPE of influenza virus-infected or PV-1-infected cells also suggests that the cytotoxic effects of caffeic acid are unlikely to result in the observed decrease in virus yields by caffeic acid.

One-step growth curve in the presence of caffeic acid. To characterize the mode of the antiviral action of caffeic acid on the multiplication of influenza A virus, a one-step growth curve was examined in the presence of $2 \mathrm{mM}$ caffeic acid. In the absence of the reagent (circle), the number of infectious progeny viruses began to increase at $3 \mathrm{~h}$ p.i. and reached the plateau at 7 to $8 \mathrm{~h}$ p.i. in the culture medium of the infected MDCK cells (Fig. 4). Under these conditions (in the absence of the reagent), CPE by the virus infection was hardly observed before $6 \mathrm{~h}$ p.i., when a small number of round cells appeared in the infected cultures. At $6 \mathrm{~h}$ p.i., the number of rounded cells increased with time, followed by the gradual detachment of the cells from the dish surface (data not shown). At $10 \mathrm{~h}$ p.i., the majority of the infected cells became rounded and only a limited fraction of the infected cells remains attached to the dish.

The addition of $2 \mathrm{mM}$ caffeic acid at the beginning of virus multiplication induced a marked decrease in the number of 

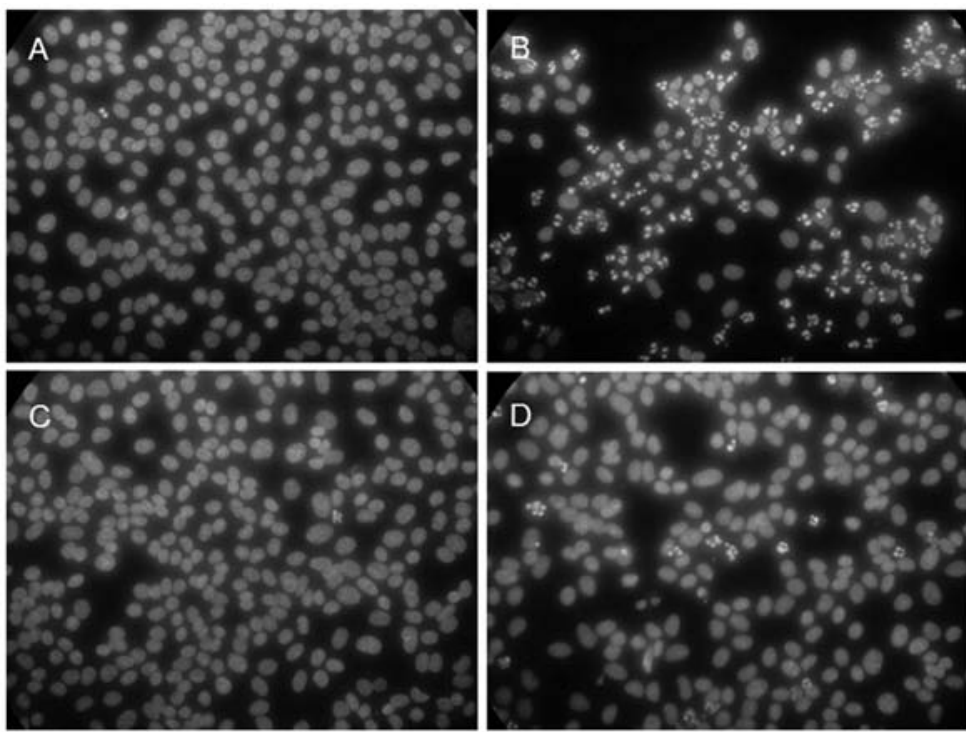

Figure 5. Effect of caffeic acid on the nuclear morphology of the infected cells. Confluent monolayers of MDCK cells were mock-infected (A and C) or infected with influenza A virus at a multiplicity of infection (MOI) of 7 (B and D). The infected cells were incubated at $37^{\circ} \mathrm{C}$ for $8 \mathrm{~h}$ in MEM containing $0.1 \%$ BSA and acetylated trypsin supplemented with (C and D) or without (A and B) $2.0 \mathrm{mM}$ caffeic acid. At $8 \mathrm{~h}$ post-infection, the cells were fixed and stained with Hoechst $\mathrm{H} 33258$ and the nuclear morphology of the cells was examined under fluorescent microscope.

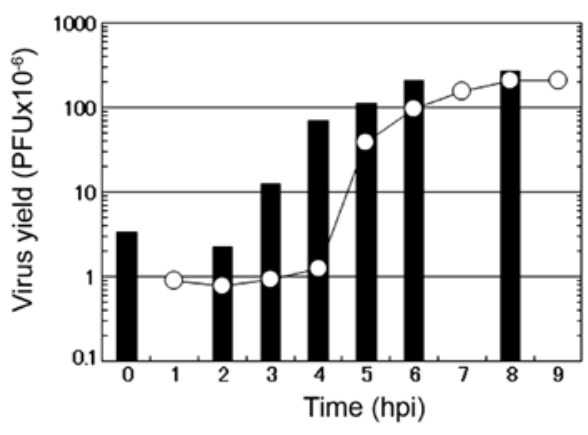

Figure 6. Effect of the time of the addition of caffeic acid on the virus yield. Two groups of confluent monolayers of MDCK cells were infected with influenza A virus at a multiplicity of infection (MOI) of 13 and incubated in Eagle's minimum essential medium (MEM) containing $0.1 \%$ bovine serum albumin (BSA) and acetylated trypsin. At the indicated time point after infection, (1) the infected cells cultured in one dish were harvested and the amount of the infectious virus was determined to reveal a one-step growth curve (open circle) and (2) the infected cells cultured in another dish received caffeic acid at the final concentration of $4.0 \mathrm{mM}$, followed by further incubation in the presence of the reagent until $9 \mathrm{~h}$ post-infection and the progeny virus in each culture was harvested to determine the amount of the final progeny virus yield in the presence of the reagent (black bar). hpi, hours post-infection.

infectious viruses in the early stage of the virus multiplication, followed by an accelerated increase in the number of the progeny viruses (Fig. 4). These results showed that the length of the eclipse period of the formation of the progeny virus in the influenza virus-infected cells was not affected by caffeic acid, although the number of infectious viruses produced in this period was greatly reduced, followed by a recovery of the formation of infectious viruses at the middle stage of the virus multiplication ( 3 to $5 \mathrm{~h}$ p.i.). These results suggest that caffeic acid mostly inhibits the multiplication of influenza virus in the early stages of viral replication in the infected MDCK cells.

In addition, the one-step growth experiment revealed that caffeic acid suppressed not only virus multiplication, but also $\mathrm{CPE}$ induced by the infection with influenza virus. The onset of CPE by viral infection was delayed in accordance with the suppression of progeny virus production; a small number of the rounded cells appeared at $8 \mathrm{~h}$ p.i. and, at $10 \mathrm{~h}$ p.i., a significant fraction (approximately 20\%) of the infected cells became rounded and the number of the detached cells was clearly lower than that in the absence of caffeic acid (data not shown).

Effect of caffeic acid on the induction of apoptosis. As described above, the presence of caffeic acid in the culture medium effectively suppressed the virus-induced cytotoxic effects in the influenza A virus-infected culture. Previously, we found that the infection with influenza virus induces apoptosis in the infected cells and compared the kinetics of the induction of apoptosis in the influenza virus-infected cells with that of the formation of progeny viruses (8). When the virus-infected cells in the absence of the reagent were fixed and stained with the DNA-binding dye, Hoechst 33258, apoptotic cell nuclei with characteristic morphology (i.e., condensation and fragmentation of cellular chromatin) appeared at $6 \mathrm{~h}$ p.i. in some (1-2\%) of the infected cells and the number of cells with these apoptotic nuclei increased with time. As shown in Fig. 5, the majority (approximately $70 \%$ or more) of the cells showed apoptotic nuclei at $8 \mathrm{~h}$ p.i. (Fig. 5B) in contrast to the mock-infected cells (Fig. 5A). In agreement with the observed suppression of the CPE by caffeic acid, the virusinduced apoptosis was greatly suppressed in the presence of the reagent; only a few (1-5\%) of the cells showed apoptotic nuclei at 8 h p.i. (Fig. 5D). Fig. 5 also shows that incubation with caffeic acid alone did not induce apoptosis (Fig. 5C), in agreement with the lack of notable cytotoxicity of the reagent.

Target of caffeic acid in the influenza virus multiplication. Hatada et al (15) reported that viral RNA replication occurs between 3 and $6 \mathrm{~h}$ p.i. in the influenza virus-infected MDCK cells and, then, the formation of progeny viruses takes place in conjunction with the envelopment of RNA nucleocapsids at 
the plasma membrane, followed by the release of the virus to the culture medium (14). In our study, the formation of progeny viruses began at approximately $4 \mathrm{~h}$ p.i. and was completed at 7 to $8 \mathrm{~h}$ p.i. under our experimental conditions (Fig. 4).

To further examine the target of the antiviral action of caffeic acid on the multiplication process of influenza virus, the 'time of addition experiment' was carried out. As shown in Fig. 6, $4 \mathrm{mM}$ caffeic acid was added to the infected culture at various time points after infection and the virus yield at the end of virus multiplication (black bar) was compared to the virus yield at the time of the addition of the reagent (open circle). The amounts of progeny virus (black bar) were markedly suppressed when the infected cells received the reagent in the early stage of the infection, such as at 0,2 or $3 \mathrm{~h}$ p.i. When the cells received caffeic acid at $3 \mathrm{~h}$ p.i., a notable degree of the suppression of the progeny virus formation was observed; however, the degree of the suppression became less and less prominent with the delay of the addition of the reagent to the infected cell culture. For example, the amount of the infectious viruses at 3 and $4 \mathrm{~h}$ p.i. was 0.95 and 1.26, respectively (open circles at 3 and $4 \mathrm{~h}$ p.i.). However, when caffeic acid was added at these time points and cell culture was continued, the final virus yields at $9 \mathrm{~h}$ p.i. were 12.4 and 70.8 , respectively, meaning that, by the addition of the reagent at $4 \mathrm{~h}$ p.i., but not at $3 \mathrm{~h}$ p.i., progeny virus formation continued significantly after the addition of the reagent. Although 70.8 was clearly less than the virus yield without the addition of caffeic acid (i.e., 207), it was far greater than the amount of the infectious virus formed at $4 \mathrm{~h}$ p.i. (i.e., 1.26). Thus, it is evident that, although the addition of caffeic acid greatly affects the progeny virus formation, it does not notably suppress the formation by the addition at $4 \mathrm{~h}$ p.i. or at a later stage.

Taking the results from the study by Hatada et al (15) into consideration, it is suggested that i) caffeic acid strongly interferes with the virus multiplication when added before the onset of RNA replication (i.e., $3 \mathrm{~h}$ p.i.); ii) it affects the formation of progeny virus to a limited extent when added after the onset of RNA replication ( $4 \mathrm{~h}$ p.i. or later). These results are in agreement with the results shown in Fig. 4 and confirm that the main target of caffeic acid is during the early stage of virus multiplication.

In this study, we demonstrate that caffeic acid effectively inhibits the multiplication of influenza A virus. This antiviral effect of caffeic acid is unlikely to be the secondary results of cytotoxicity of the reagent or the direct inactivation of the virus (virucidal effect), but is more likely due to the specific interaction of caffeic acid to cellular and/or viral proteins involved in viral genome replication processes. Both the results in Figs. 4 and 6 reveal that caffeic acid mainly interferes with the multiplication of influenza A virus in the early stage of viral multiplication, possibly at the step(s) for a preparation of the replication of viral genome RNA in the infected cells. Caffeic acid has been known to directly interact with proteins. For example, Kang et al (16) found a specific binding of caffeic acid to Fyn kinase, one of the members of the non-receptor protein kinase family, resulting an inhibition of the enzyme activity and Trnkova et al (17) also examined a binding constant of caffeic acid to bovine serum albumin. These direct interactions between caffeic acid and proteins suggest that caffeic acid inhibits virus multiplication by directly binding and interacting with certain enzyme(s) necessary for preparing viral RNA replication in influenza virus-infected cells.
In addition, the results of our study on the morphological alteration of the infected cells and cell nuclei (Fig. 5) revealed that, in addition to the inhibition of virus multiplication, caffeic acid suppressed the induction of cytopathogenic changes of the infected cells, such as CPE and apoptosis, confirming the weak cytotoxicity of the reagent. The observed antiviral activity without notable cytotoxicity supports a potential application of caffeic acid or its derivatives as an anti-influenza viral drug.

\section{Acknowledgements}

The authors thank Dr Tsutomu Arakawa for his stimulative discussions and assistance with the editing of the manuscript. This study was supported in part by research grants from All Japan Coffee Association.

\section{References}

1. Uozaki M, Yamasaki H, Katsuyama Y, Higuchi M, Higuchi T and Koyama AH: Antiviral effect of octyl gallate against DNA and RNA viruses. Antiviral Res 73: 85-91, 2007.

2. Utsunomiya $H$, Ichinose M, Uozaki M, Tsujimoto K, Yamasaki H and Koyama AH: Antiviral activities of coffee extracts in vitro. Food Chem Toxicol 46: 1919-1924, 2008.

3. Uozaki M, Ikeda K, Tsujimoto K, Nishide M, Yamasaki H, Khamsri B and Koyama AH: Antiviral effects of dehydroascorbic acid. Exp Ther Med 1: 983-986, 2010.

4. Arakawa T, Yamasaki H, Ikeda K, Ejima D, Naito T and Koyama AH: Antiviral and virucidal activities of natural products. Curr Med Chem 16: 2485-2497, 2009.

5. Ikeda K, Tsujimoto K, Uozaki M, Nishide M, Suzuki Y,Koyama AH and Yamasaki H: Inhibition of multiplication of herpes simplex virus by caffeic acid. Int J Mol Med 28: 595-598, 2011.

6. Rice-Evans CA, Miller NJ and Paganga G: Structure-antioxidant activity relationships of flavonoids and phenolic acids. Free Radic Biol Med 20: 933-956, 1996.

7. Koyama AH and Uchida T: The effect of ammonium chloride on the multiplication of herpes simplex virus type 1 in Vero cells. Virus Res 13: 271-282, 1989.

8. Kurokawa M, Koyama AH, Yasuoka S and Adachi A: Influenza virus overcomes apoptosis by rapid multiplication. Int $\mathbf{J}$ Mol Med 3: 527-530, 1999.

9. Koyama AH, Irie H, Ueno F, Ogawa M, Nomoto A and Adachi A: Suppression of apoptotic and necrotic cell death by poliovirus. J Gen Virol 82: 2965-2972, 2001.

10. McGarrity GJ: Detection of contamination. Methods Enzymol 58: 18-29, 1979.

11. Murayama M, Tsujimoto K, Uozaki M, Katsuyama Y, Yamasaki H, Utsunomiya $\mathrm{H}$ and Koyama AH: Effect of caffeine on the multiplication of DNA and RNA viruses. Mol Med Rep 1: 251-255, 2008.

12. Roizman B and Knipe DM: Herpes simplex virus and their replication. In: Fields Virology. Fields BN, Knipe DM and Howley PM (eds). 4th edition, Lippincott-Raven, New York, pp2399-2460, 2001.

13. Racaniello VR: Picornaviridae: The viruses and their replication. In: Fields Virology. Fields BN, Knipe DM and Howley PM (eds). 4th edition, Lippincott-Raven, Philadelphia, pp685-722, 2001.

14. Lamb RA and Kruchikokug RM: Orthomyxoviridae: The viruses and their replication. In: Fields Virology. Fields BN, Knipe DM and Howley PM (eds). 4th edition, Lippincott-Raven, Philadelphia, pp1487-1530, 2001.

15. Hatada E, Hasegawa M, Mukaigawa J, Shimizu K and Fukuda R: Control of influenza virus gene expression: quanatitative analysis of each viral RNA species in infected cells. J Biochem 105: 537-546, 1989.

16. Kang NJ, Lee KW, Shin BJ, Jung SK, Hwang MK, Bode AM, Heo YS, Lee HJ and Dong Z: Caffeic acid, a phenolic phytochemical in coffee, directly inhibits Fyn kinase activity and UVB-induced COX-2 expression. Carcinogenesis 30: 321-330, 2009.

17. Trnkova L, Bousova I, Kubicek V and Drsata J: Binding of naturally occurring hydroxycinnamic acids to bovine serum albumin. Nat Sci 2: 563-570, 2010. 